\title{
Primary Sarcomatoid Malignant Pericardial Mesothelioma
}

\author{
Daming Jiang ${ }^{1}$, Minjian Kong ${ }^{1},{\mathrm{Jie} \mathrm{Li}^{2} \text { and Jianfang Qian }}^{1}$
}

Key words: sarcomatoid malignant mesothelioma, pericardium

(Intern Med 52: 157-158, 2013)

(DOI: 10.2169/internalmedicine.52.8934)
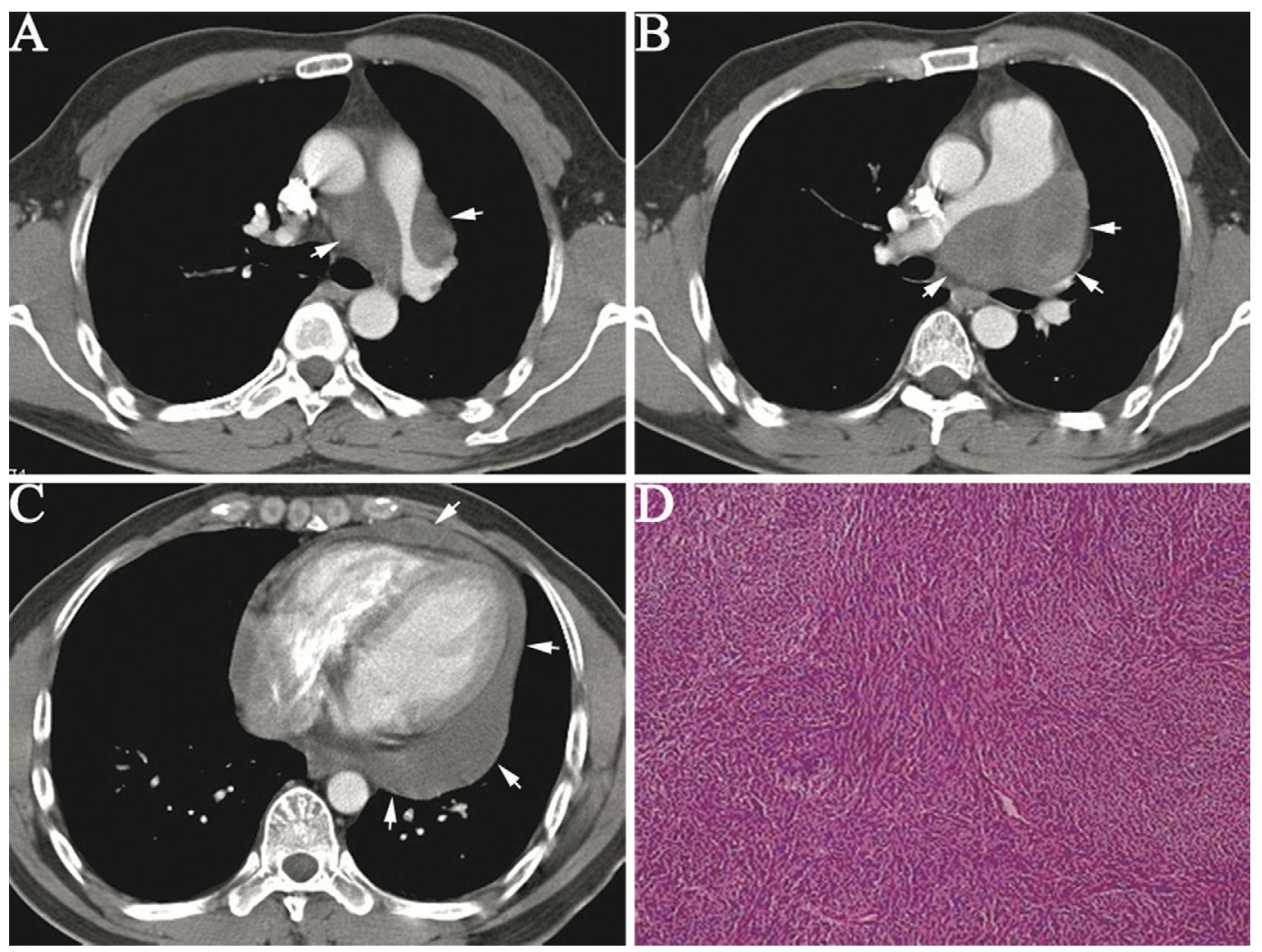

Picture.

A 39-year-old man was admitted to our hospital with a 10-month history of shortness of breath and chest distress on exertion. Echocardiography showed a pericardial mass and effusion. Computed tomography (CT) (Picture A, B) demonstrated a thickened pericardium, effusion and a large pericardial mass (arrows) encompassing the left pulmonary artery compressing the left and right pulmonary arteries (Picture C). A positron emission tomography (PET) scan was positive along the pericardium. A pericardial biopsy using video-assisted thoracic surgery confirmed a diagnosis of sarcomatoid malignant mesothelioma (Picture D, Hematox- ylin and Eosin staining, $\times 20$ ). Immunohistochemical staining for calretinin, vimentin and CD34 was positive, while staining for EMA, SMA, desmin and S100 protein was negative. Owing to the sensitive location and poor prognosis of the tumor, the patient received no radiation treatment or chemotherapy. During follow-up, the symptoms of cough and shortness of breath worsened, and repeated CT revealed tumor progression. The patient died of cardiac failure 11 months after the diagnosis was made. Primary malignant pericardial mesothelioma (PMPM) is extremely rare, with an incidence of $0.0022 \%$ in an autopsy series of 500,000 case

${ }^{1}$ Department of Heart Center, 2nd Affiliated Hospital, School of Medicine, Zhejiang University, China and ${ }^{2}$ Guangdong Cardiovascular Institute, Guangdong General Hospital, Guangdong Academy of Medical Science, China

Received for publication September 8, 2012; Accepted for publication September 30, 2012

Correspondence to Dr. Jianfang Qian, cardioqjf@163.com 
studies (1). There are four histological types of PMPM, including epithelioid, biphasic, desmoplastic and sarcomatoid, among which the sarcomatoid type is very rare $(2,3)$. The clinical presentation can include constrictive pericarditis, pericardial effusion, cardiac tamponade and heart failure. Making a diagnosis of PMPM is difficult, and most cases are only diagnosed at autopsy. CT and magnetic resonance imaging are useful, and PET-CT can help to detect distant metastases. Surgical intervention remains controversial, however, it may be curative in localized cases. Few patients benefit from radiation treatment or chemotherapy. The prognosis of PMPM is very poor, and most patients die within one year of being diagnosed (1-3).
The authors state that they have no Conflict of Interest (COI).

\section{References}

1. Lee MJ, Kim DH, Kwan J, et al. A case of malignant pericardial mesothelioma with constrictive pericarditis physiology misdiagnosed as pericardial metastatic cancer. Korean Circ J 41: 338-341, 2011.

2. Reardon KA, Reardon MA, Moskaluk CA, Grosh WW, Read PW. Primary pericardial malignant mesothelioma and response to radiation therapy. Rare Tumors 2: e51, 2010.

3. Terada T. Primary sarcomatoid malignant mesothelioma of the pericardium. Med Oncol 29: 1345-1346, 2012.

(C) 2013 The Japanese Society of Internal Medicine http://www.naika.or.jp/imonline/index.html 